\title{
Anti-CD3/MUC1 Antibody-armed PD-1 Inhibitor-induced Cytokine-induced Killer Cells
}

National Cancer Institute

\section{Source}

National Cancer Institute. Anti-CD3/MUC1 Antibody-armed PD-1 Inhibitor-induced

Cytokine-induced Killer Cells. NCI Thesaurus. Code C148543.

A preparation of cytokine-induced killer cells (CIKs), which have been exposed, ex vivo, to a specific set of cytokines and a programmed cell death protein 1 (PD-1) inhibitor, mixed with a bispecific anti-cluster of differentiation 3 (CD3)/anti-mucin-1 (MUC1) antibody, with potential anti-tumor cytotoxic activity. Upon administration of the anti-CD3/MUC1 antibody-armed PD-1 inhibitor-induced CIKs, the antibody moiety binds to both CD3 on the CIKs and MUC1 on cancer cells. This crosslinks the CIKs and tumor cells, which allows the CIKs to target and lyse MUC1-expressing cancer cells. PD-1 blockade activates the CIKs. The cytokines used, usually interferon-gamma (IFNg), interleukin 1 (IL-1), and IL-2, stimulate the proliferation and maturation of peripheral blood mononuclear cells (PBMCs) into CIK cells. Anti-CD3 stimulation allows for the CIKs' improved lytic activity. 